\title{
Retroperitoneal Approach for Lower Extremity Arteriovenous Graft as Long-Term Vascular Access in a Complex Case
}

\author{
Mingli Zhu ${ }^{\mathrm{a}}$ Yaxue Shi $^{\mathrm{b}}$ Jiejun Cheng ${ }^{\mathrm{c}}$ Huihua Pang ${ }^{\mathrm{a}}$ \\ Leyi Gu $^{\mathrm{a}}$ Aiwu Lin ${ }^{\mathrm{a}}$ Ying Huang ${ }^{\mathrm{a}}$ Yucheng Yan ${ }^{\mathrm{a}}$ \\ ${ }^{a}$ Renal Division, ${ }^{b}$ Vascular Surgery Department, and ${ }^{c}$ Radiology Department, \\ Renji Hospital, Shanghai Jiaotong University School of Medicine, \\ Shanghai, China
}

\section{Key Words}

Hemodialysis · Vascular access · Arteriovenous graft · Retroperitoneal approach

\begin{abstract}
A 76-year-old woman with stage 5 chronic kidney disease requiring hemodialysis had a long history of hypertension and diabetes. Her venous access was exhausted because of several previous access procedures including autogenous arteriovenous fistula operations and central venous catheter placements. After evaluating the function of her vessels when she was transferred to Renji hospital, we created an arteriovenous graft via a retroperitoneal approach in her right lower extremity. We inserted two grafts into her right thigh, a straight external ring and a loop graft. Twenty days after operation the patient's new access was successfully used in the treatment of hemodialysis. She has been followed up to now without occurrence of any graft-related events.
\end{abstract}

\section{Case Presentation}

A 76-year-old woman with end-stage renal disease due to hypertensive nephrosclerosis had been undergoing renal replacement therapy for 10 years. She also had an 8-year history of type II diabetes that was treated with diet control and without hypoglycemic medicine. She accepted 7 treatments of hemodialysis because of acute renal failure 27 years ago. Radial artery puncture was performed as temporary vascular access in hemodialysis. When she was 66 years old, she accepted temporary hemodialysis with radial artery puncture before she started chronic peritoneal dialysis due to stage 5 chronic kidney disease. After treatment with peritoneal dialysis for 5 years, she had to switch to hemodialysis as a result of severe peritonitis until now. The patient received 6 temporary catheter placements due to autogenous arteriovenous fistula (AVF) failure during 5 years. Her right and left subclavian veins, and her right and left femoral veins had been cannulated with temporary or 
long-term catheters. She was transferred to Renji Hospital with a non-functioning long-term double-lumen catheter in her left femoral vein.

A venogram demonstrated that her bilateral upper limb veins, including the brachiocephalic and subclavian veins, were occlusive. We also discovered a bilateral occlusion of the external iliac veins of the lower limbs. The only unobstructed central vein was the right common iliac vein. An angiogram of the left femoral vein was conducted by injecting contrast agent into the catheter and showed that the catheter was inserted in a branch of the iliac vein and the main line was occlusive. We decided to use an atypical approach to place a graft into the patient's lower extremity. We inserted a straight external ring graft (GORE-TEX ${ }^{\oplus}, 6 \times 100 \mathrm{~mm}$ ) into the right common iliac vein by end-to-side anastomosis (diameter: $1.5 \mathrm{~cm}$ ) via a retroperitoneal approach, and then tunneled the graft under the inguinal ligament to the mid-superficial tissues with the anterior thigh. Another subcutaneous graft, a loop graft (GORE-TEX, $6 \times 400 \mathrm{~mm}$ ), was implanted to connect the common femoral artery and the previous straight graft in the right thigh; the anastomotic diameter of the artery was $0.8 \mathrm{~cm}$. After the operation, we observed adequate thrill on the arteriovenous graft (AVG) (i․, 1).

The graft was successfully applied 20 days after operation. The graft has been used in hemodialysis up to now for nearly 8 months without any graft-related event or discomfort. The blood flow has remained $250 \mathrm{ml} / \mathrm{min}$.

\section{Discussion}

A well-functioning vascular access remains a key issue of hemodialysis and is essential to providing efficient dialysis therapy. We described a complex case of vascular access. All of the patient's venous accesses were exhausted so that we were unable to create a new vascular access. The patient had a long history of hypertension, diabetes and dialysis. She underwent several previous access procedures including AVF operations and central venous catheter placements. An autogenous AVF is preferred and generally recommended $[1,2]$. However, it is not always the ideal choice for certain subsets of patients. This patient with advanced age and diabetes may have a limited number of suitable sites for the formation of primary AVF [3]. Catheters are necessary to provide temporary extracorporeal vascular access to dialysis for uremia patients in whom arteriovenous access may be problematic, impossible or delayed. The present patient had received more than 6 catheter placements for dialysis. Her available central veins had all been exhausted.

Prosthetic AVGs usually are indicated in patients with AVF failure, exhausted superficial veins or unsuitable vessels and are a better alternative for patients than catheters $[4,5]$. In light of the ever increasing number of patients with end-stage renal disease, the ageing dialysis population and their prolonged longevity, surgeons increasingly encounter difficult access problems, such as unavailable upper extremity access sites and central venous outflow obstruction resulting from previous catheterization. Therefore, increasing complex arteriovenous bypass grafts including a lower extremity graft is reserved for patients with the above-mentioned characteristics. Antoniou et al. [6] identified 3 main types of lower extremity vascular access: upper thigh prosthetic, mid-thigh prosthetic and femoral vein transposition arteriovenous access. According to access procedures, examinations and images, the present patient's vessels were unsuitable for the creation of a vascular access. We had to place an AVG between the right femoral artery and right common iliac vein. Two grafts were used, one was connected to the common iliac vein, and the other was connected to the femoral artery; these two grafts were anastomosed by end-to-end technique. The retroperitoneal approach for lower extremity AVG is a novel way to 
create vascular access for maintenance dialysis. Recently, Colvard et al. [7] showed this technique in two cases in their center. This new approach may provide chances for some patients to continue dialysis. By using this kind of access, they may have advantages in the treatment of hemodialysis, such as convenient puncture, sufficient blood flow and good adequacy. However, the limitations of this kind of access include the difficulty of achieving hemostasis, uneasy run for movement. If the GORE-TEX graft gets infected, we have to deal with serious complications. Therefore, the retroperitoneal approach for lower extremity AVG should be recommended only for patients without available arteriovenous access.

Nephrologists and surgeons should work jointly to pinpoint the appropriate timing of access and to allow adequate time for either fistula maturation or graft placement [8]. Late referral of patients to nephrologists and surgeons limits the use of AVFs. An early graft is the superior option to a central venous catheter for vascular access when a primary AVF is impossible. Central venous catheters have many harmful complications and may limit the future availability of access sites because of the development of central venous stenosis [9]. An impaired venous outflow due to stenosis precludes the creation of any vascular access [10]. Patients with central venous catheter placement history should have their central veins evaluated by interventional digital subtraction angiography. Long-term dialysis catheters should be avoided, particularly on the same side of a maturing venous access. A comprehensive review of previous access procedures and full physical examination with vessel mapping is necessary before the insertion of a prosthetic AVG.

In summary, dialysis-dependent patients undoubtedly need a multidisciplinary team to manage the multiple issues related to the creation and maintenance of dialysis accesses. The choice of dialysis access should be individualized and based on which access will provide lifetime maximum utilization. AVGs and other forms of vascular access should be regarded as providing complimentary roles in vascular access provision.

\section{Disclosure Statement}

The authors have no conflicts of interest to disclose. 


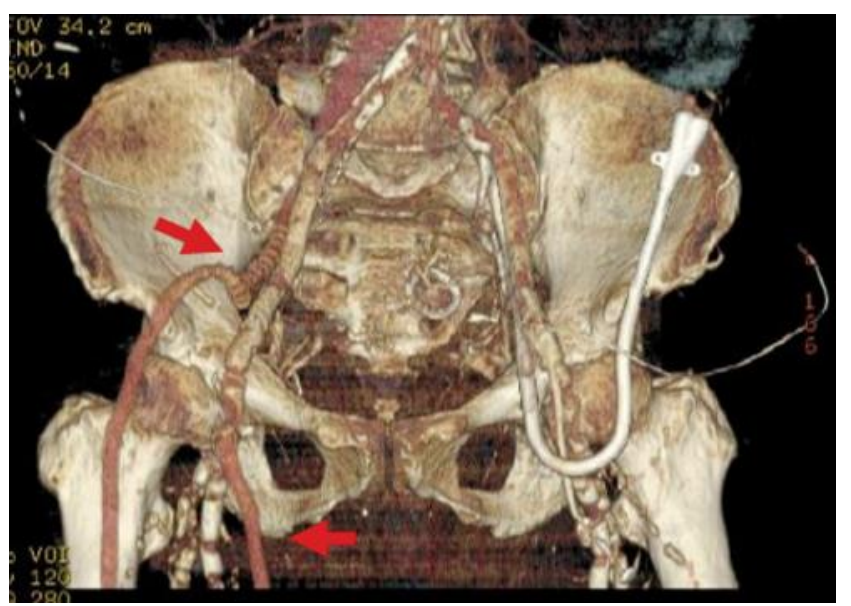

Fig. 1. CAT scan of the pelvic cavity illustrates the retroperitoneal approach for right femoral artery and right common iliac vein AVG. The red arrow to the left indicates the straight external ring graft, the red arrow to the right indicates the subcutaneous loop graft.

\section{References}

1 Vascular Access Work Group: Clinical practice guidelines for vascular access. Am J Kidney Dis 2006;48(suppl 1):S248-S272.

-2 Tordoir J, Canaud B, Haage P, Konner K, Basci A, Fouque D, Kooman J, Martin-Malo A, Pedrini L, Pizzarelli F, Tattersall J, Vennegoor M, Wanner C, ter Wee P, Vanholder R: EBPG on vascular access. Nephrol Dial Transplant 2007;22(suppl 2):ii88-ii117.

3 Teixeira JF: Native AV fistula - How far should we go? - Not so far. Paper presented at: 3rd International Congress of the Vascular Access Society (VAS); May 21-23, 2003; Lisbon, Portugal. Blood Purif 2003;21:417-451.

4 Akoh JA: Prosthetic arteriovenous grafts for hemodialysis. J Vasc Access 2009;10:137-147.

5 Ethier J, Mendelssohn DC, Elder SJ, Hasegawa T, Akizawa T, Akiba T, Canaud BJ, Pisoni RL: Vascular access use and outcomes: an international perspective from the Dialysis Outcomes and Practice Patterns Study. Nephrol Dial Transplant 2008;23:3219-3226.

-6 Antoniou GA, Lazarides MK, Georgiadis GS, Sfyroeras GS, Nikolopoulos ES, Giannoukas AD: Lower-extremity arteriovenous access for haemodialysis: a systematic review. Eur J Vasc Endovasc Surg 2009;38:365-372.

7 Colvard BD, Anaya-Ayala JE, Palacios-Reyes D, Cheema ZF, Smolock CJ, Davies MG, Peden EK: Increasing dialysis access options in lower extremity: retroperitoneal approach for external iliac artery-vein arteriovenous graft. J Vasc Access 2011;12:365-368.

8 Schild AF: Maintaining vascular access: the management of hemodialysis arteriovenous grafts. J Vasc Access 2010;11:92-99.

-9 Thomson PC, Stirling CM, Geddes CC, Morris ST, Mactier RA: Vascular access in haemodialysis patients: a modifiable risk factor for bacteraemia and death. QJM 2007;100:415-422.

10 Berardinelli L: The endless history of vascular access: a surgeon's perspective. J Vasc Access 2006;7:103-111. 